\title{
One-trial learning in larval Drosophila
}

\author{
Aliće Weiglein, ${ }^{1}$ Florian Gerstner, ${ }^{1,2}$ Nino Mancini, ${ }^{1}$ Michael Schleyer, ${ }^{1}$ \\ and Bertram Gerber ${ }^{1,3,4}$
}

\author{
${ }^{1}$ Department of Genetics, Leibniz Institute for Neurobiology, 39118 Magdeburg, Germany; ${ }^{2}$ Department of Animal Physiology, \\ University Bayreuth, 95447 Bayreuth, Germany; ${ }^{3}$ Institute of Biology, Otto von Guericke University Magdeburg, 39106 Magdeburg, \\ Germany; ${ }^{4}$ Center for Behavioral Brain Sciences, 39106 Magdeburg, Germany
}

\begin{abstract}
Animals of many species are capable of "small data" learning, that is, of learning without repetition. Here we introduce larval Drosophila melanogaster as a relatively simple study case for such one-trial learning. Using odor-food associative conditioning, we first show that a sugar that is both sweet and nutritious (fructose) and sugars that are only sweet (arabinose) or only nutritious (sorbitol) all support appetitive one-trial learning. The same is the case for the optogenetic activation of a subset of dopaminergic neurons innervating the mushroom body, the memory center of the insects. In contrast, no onetrial learning is observed for an amino acid reward (aspartic acid). As regards the aversive domain, one-trial learning is demonstrated for high-concentration sodium chloride, but is not observed for a bitter tastant (quinine). Second, we provide follow-up, parametric analyses of odor-fructose learning. Specifically, we ascertain its dependency on the number and duration of training trials, the requirements for the behavioral expression of one-trial odor-fructose memory, its temporal stability, and the feasibility of one-trial differential conditioning. Our results set the stage for a neurogenetic analysis of one-trial learning and define the requirements for modeling mnemonic processes in the larva.
\end{abstract}

[Supplemental material is available for this article.]

For all animals, it is beneficial to learn predictors of where and when rewards such as nutrients can be found, or under which conditions threats such as predators, injury, or toxic food need to be reckoned with. This is because such predictive learning allows the anticipatory control of behavior (Hoffmann et al. 2007). In this context, onetrial associative learning is an interesting study case, since a single co-occurrence of events is actually of little predictive value. Nevertheless, in biological learning systems one-trial associative learning has been observed in a number of species and across valence domains (e.g., zebrafish: Blank et al. 2009; chicken: Cherkin 1969; quail: Hilliard et al. 1997; mice: Abt et al. 1961; rats: Cammarota et al. 2005; Wood et al. 2004; humans: Haesen et al. 2017), notably including insects. For example, honeybees show single-trial learning of odors as predictors of sugar-water (Takeda 1961; for review, see Giurfa and Sandoz 2012) or of electroshock punishment (Vergoz et al. 2007; for review, see Tedjakumala and Giurfa 2013). In adult Drosophila, a single trial of odor preceding electroshock punishment or sugar reward can establish aversive or appetitive associative memory, respectively (Tully and Quinn 1985; Beck et al. 2000; Colomb et al. 2009; Scheunemann et al. 2013). In addition, onetrial learning can be demonstrated upon pairing odor with the optogenetic activation of subsets of dopaminergic neurons innervating the mushroom body in adult flies (Aso and Rubin 2016; König et al. 2018).

Larval Drosophila has recently emerged as an analytically potent study case for understanding the neurogenetics of associative learning (for reviews, see Gerber and Stocker 2007; Diegelmann et al. 2013; Thum and Gerber 2018). This is thanks to a fortunate combination of learning ability, neural simplicity in terms of cell numbers, tractability for synapse-resolution connectomics, and the availability of both a light-microscopy atlas of its neurons and the genetic toolbox available for manipulating them one at a

Corresponding authors: Alice.Weiglein@lin-magdeburg.de; Bertram. Gerber@lin-magdeburg.de

Article is online at http://www.learnmem.org/cgi/doi/10.1101//m.049106.118. time (Duffy 2002; Venken et al. 2011; Li et al. 2014; Housden and Perrimon 2016). Two kinds of paradigm for odor-taste associative learning in the larva are commonly used, namely absolute and differential conditioning. In absolute conditioning, separate experimental groups of larvae are trained to associate an odor with either the presence or the absence of a reward such as fructose, for example, and are then tested for their odor preference. In differential conditioning, one odor is paired with reward whereas a second odor is presented without reward (the chemical identity of the odors is alternated between experimental groups); in a subsequent test, the animals are given the choice between the two odors. In neither case, however, have systematic analyses of one-trial associative learning been reported. Rather, analyses of associative learning in the larva have so far focused almost exclusively on three-trial conditioning, whether for various sugars (Scherer et al. 2003; Schipanski et al. 2008; Rohwedder et al. 2012) and amino acids as taste rewards (Schleyer et al. 2015a), for optogenetically induced reward learning through the activation of subsets of dopaminergic neurons (Saumweber et al. 2018), for punishment by substrate vibration (Eschbach et al. 2011; Saumweber et al. 2014) or electric shocks (Pauls et al. 2010), or for quinine as taste punishment (Gerber and Hendel 2006; El-Keredy et al. 2012; Apostolopoulou et al. 2014). Only for salt, that is high concentrations of sodium chloride, have analyses of three-trial learning (Gerber and Hendel 2006; Niewalda et al. 2008) recently been complemented by an experiment reporting associative memory after just one training trial (Widmann et al. 2016; loc. cit. Fig. 7A).

The present study systematically tests for one-trial associative learning using fructose, arabinose, sorbitol, and aspartic acid as

(C) 2019 Weiglein et al. This article is distributed exclusively by Cold Spring Harbor Laboratory Press for the first 12 months after the full-issue publication date (see http://learnmem.cshlp.org/site/misc/terms.xhtml). After 12 months, it is available under a Creative Commons License (Attribution-NonCommercial 4.0 International), as described at http://creativecommons.org/licenses/by-nc/ $4.0 \%$. 
taste rewards, for optogenetic activation of the dopaminergic DAN-i1 neuron as a reward signal, and for high-concentration salt and quinine as taste punishment. Focusing on fructose, we further provide key parametric features of one-trial learning.

\section{Results}

One-trial memory is detectable for all sugar types, but not for aspartic acid as reward

Larvae were trained in the absolute conditioning paradigm such that for one experimental group the animals received the odor $n$-amylacetate together with a taste reward (paired training group), whereas in a second experimental group the larvae received the odor and the reward unpaired from one another (unpaired training group). The difference in odor preference between paired-trained and unpairedtrained animals thus reflects associative memory and is quantified as the performance index (PI). Positive and negative PI scores indicate appetitive and aversive associative memory scores, respectively. We observed appetitive memory after only one such training trial with fructose (Fig. 1A), arabinose (Fig. 1B), and sorbitol (Fig. 1C), but not with aspartic acid (Fig. 1D). Specifically, for fructose, arabinose and sorbitol memory scores increased with more prolonged training trial durations, reaching asymptotic levels with training trial durations of $\sim 2-4 \mathrm{~min}$; for aspartic acid, memory scores were uniformly low (given a nonsignificant Kruskal-Wallis test $(P=0.45)$, we refrain from discussing possible trends for the different training trial durations using aspartic acid) (Fig. 1E). Using three training trials, and mostly two-odor differential conditioning, appetitive memory has previously been observed for all these tastant rewards (fructose: Scherer et al. 2003; Saumweber et al. 2011; arabinose and sorbitol: Rohwedder et al. 2012; aspartic acid: Schleyer et al. 2015a).

\section{One-trial memory is detectable for high-concentration salt but not for quinine as punishment}

Using three training trials, and mostly two-odor differential conditioning, aversive memory has previously been observed for both high-concentration salt and for quinine (high-concentration salt: Gerber and Hendel 2006; Niewalda et al. 2008; Widmann et al. 2016; quinine: Gerber and Hendel 2006; Schleyer et al. 2011; El-Keredy et al. 2012). In both cases, the behavioral expression of
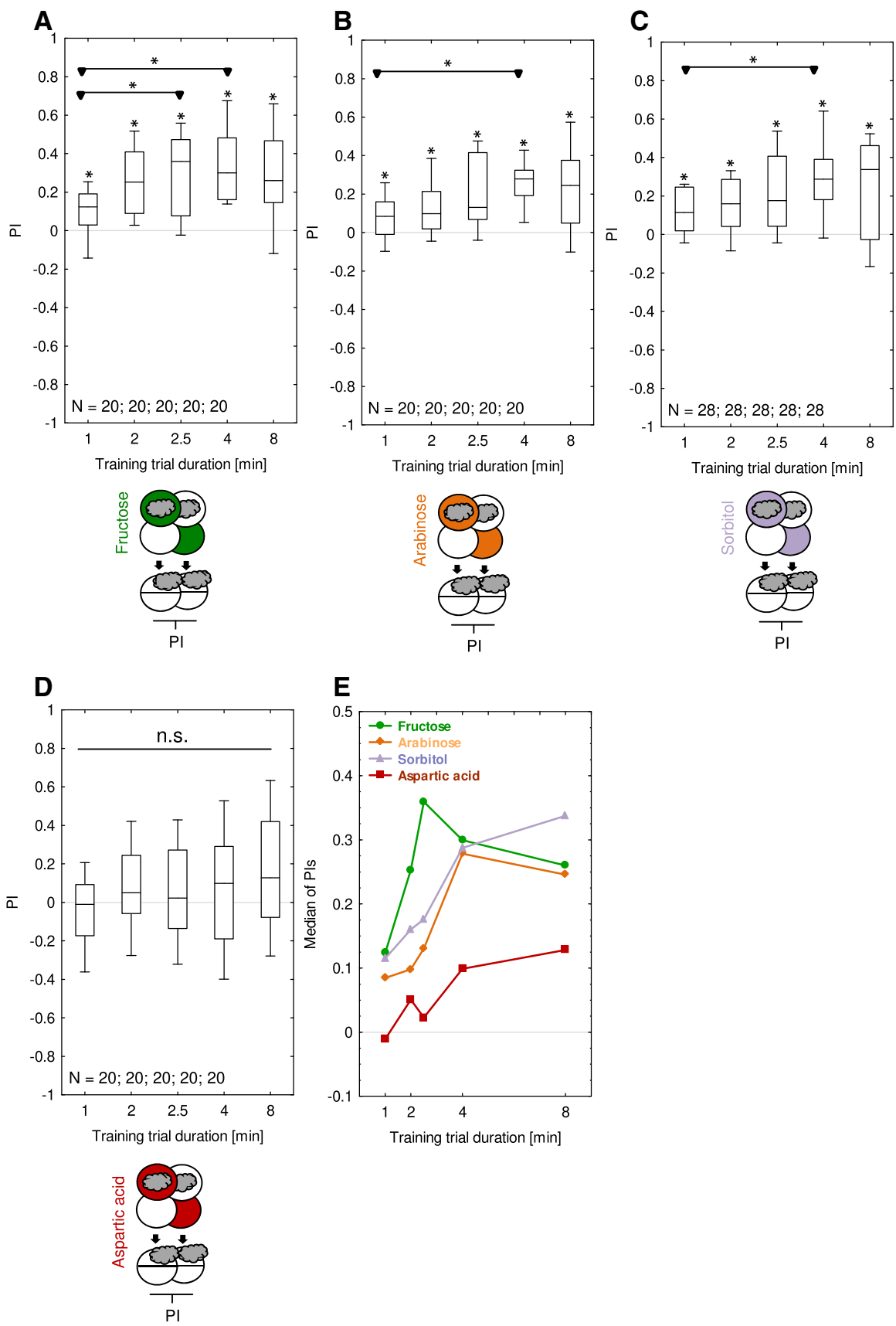

Figure 1. One-trial associative memory is detectable for sugars, but not for aspartic acid reward. Larvae underwent single training trials of 1,2, 2.5, 4, or 8 min duration, with odor and the respective taste reward either together (Paired) or separate (Unpaired). Three different kinds of sugar or aspartic acid were used as a reward, as shown toward the bottom of the figures in the sketches of the training and testing procedures. Green, orange, light purple, and red Petri dishes represent fructose, arabinose, sorbitol, and aspartic acid, respectively; dishes without fill indicate Petri dishes with only the substrate, that is pure agarose, but without any tastant added. The gray cloud indicates the odor $n$-amylacetate. Throughout this study, the sequence of events during training was as depicted in half of the cases, whereas for the other half it was reversed (not shown). Differences in odor preference after paired versus unpaired training are quantified by the $\mathrm{PI}$ and thus reflect associative memory. $\mathrm{Pls}>0$ indicate appetitive memory, $\mathrm{Pls}<0$ aversive memory. Pls were positive for all training trial durations and increased with longer training trial durations for $(A)$ fructose, $(B)$ arabinose, and $(C)$ sorbitol. For $(D)$ aspartic acid, no appetitive memory was detectable. Asterisks above box plots reflect significance at $P<0.05$ in OSS-tests with Bonferroni-Holm (BH) correction. A Kruskal-Wallis (KW) test across all experimental conditions is significant at $P<0.05$ for all sugar types but not for aspartic acid. Asterisks above horizontal lines reflect significance at $P<0.05$ in MWU-tests with $\mathrm{BH}$ correction. Data are displayed as box plots, with the median as the middle line, the box boundaries as $25 \%$ and $75 \%$ quantiles, and the whiskers as $10 \%$ and $90 \%$ quantiles. Sample sizes are indicated within the figure. Preference scores underlying the Pls are documented in Supplemental Figure S1. (E) The medians of the respective PIs from $(A-D)$ are shown across training trial duration. 
aversive memory is best grasped as a form of escape because it requires the presence of the punishment during the test to motivate learned avoidance (innate olfactory behavior is not affected by the presence of either tastant: Gerber and Hendel 2006; Schleyer et al. 2011). Here we show that high-concentration salt (1.5 M sodium chloride) is also effective as a punishment in our one-trial paradigm. Aversive memory scores were significantly negative after one training trial of 4 and 8 min duration (Fig. 2A); a trend toward appetitive (sic) memory after $1 \mathrm{~min}$ training did not reach significance (OSS: $P=0.02$, which is above the $\mathrm{BH}$ corrected significance threshold of 0.0167$)$. For quinine, using only one training trial in our paradigm did not yield significant aversive memory. Since the Kruskal-Wallis test across training trial durations was not significant $(P=0.2)$ we refrain from discussing possible trends (Fig. 2B,C).

\section{Optogenetic activation of 864-DAN has a rewarding effect in the one-trial paradigm}

We further investigated whether optogenetic activation of DAN-i1, as covered in the 864-DAN driver strain, would have a rewarding effect in our one-trial paradigm (for three training trials see Saumweber et al. 2018). Indeed, activation of 864-DAN at the times when otherwise a tastant reinforcer would have been presented resulted in appetitive memory scores in the experimental genotype; memory scores were significantly different from zero and from both genetic controls (Fig. 3A). The expression pattern of the
864-DAN driver strain crossed to the ChR2-XXL effector strain was confirmed by immunohistochemistry against ChR2-XXL as the effector protein (Fig. 3B). The hemispherically unique DAN-i1 neurons each innervate the upper toe of the medial lobe of the mushroom body of each brain hemisphere (Fig. 3C) (Saumweber et al. 2018).

On the basis of the above results, we decided to investigate one-trial fructose memory further. We chose fructose both because fructose yielded the highest scores, in particular for relatively short training trial durations (Fig. 1E), and because fructose has been used the most in previous multiple-trial studies on the mechanisms of learning in the larva.

\section{One-trial fructose memory is behaviorally expressed in the absence but not in the presence of fructose}

For fructose memory after three training trials, it has been reported that memory is not behaviorally expressed if the test is carried out in the presence of fructose (Gerber and Hendel 2006; Schleyer et al. 2011, 2015a). Arguably, this is because after odor-fructose associative learning the larvae track down the odor in search of fructose, a behavior that is no longer adaptive as soon as the sought-for fructose reward is found (innate olfactory behavior is not affected by the presence of fructose: Gerber and Hendel 2006; Schleyer et al. $2011,2015 a)$. We observed the same effect for one-trial fructose memory: larvae behaviorally expressed fructose memory when tested in the absence, but not when tested in the presence of fructose (Fig. 4). We next sought to ascertain whether, using the present paradigm, memory scores further increased when more training trials were used.
A
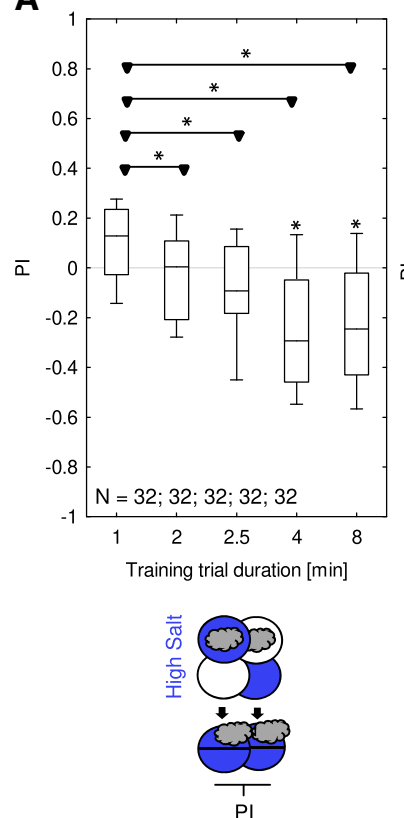

B
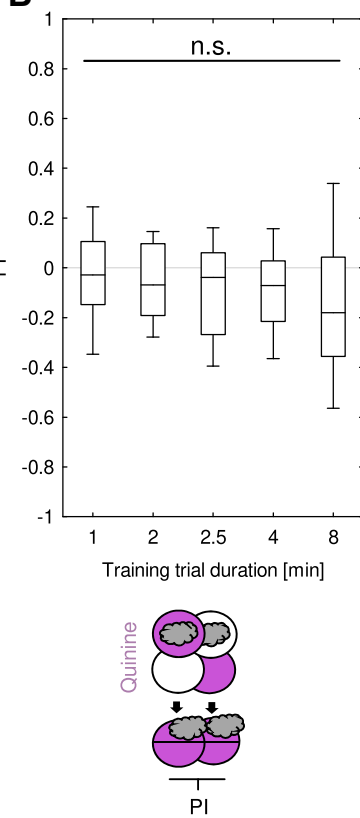

C

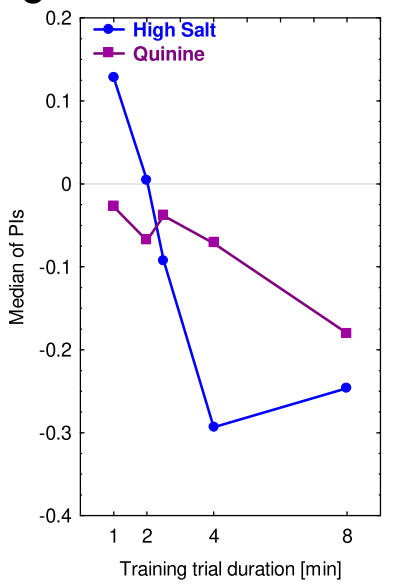

Figure 2. One-trial associative memory is detectable for high-concentration salt but not for quinine as punishment. Larvae underwent single training trials of 1, 2, 2.5, 4, or 8 min duration, with odor and the respective taste punishment either together (Paired) or separate (Unpaired). Differences in odor preference after such training are quantified by the $\mathrm{PI}$ and reflect associative memory. Two different taste punishments were used, as shown toward the bottom of the figures in the sketches of the training and testing procedures. Blue and magenta Petri dishes indicate high-concentration salt and quinine, respectively; dishes without fill indicate Petri dishes with only the substrate, that is pure agarose, but without any tastant added. The gray cloud indicates the odor $n$-amylacetate. Aversive memory scores were observed when testing in the presence of $(A)$ high-concentration salt for the longer training trial durations. When testing in the presence of $(B)$ quinine no aversive memory could be detected. Other details as described in Figure 1. Preference scores underlying the Pls are documented in Supplemental Figure S2. (C) The medians of the respective PIs from $(A, B)$ are shown across training trial duration.

\section{Fructose memory scores increase with training trial number}

For two-odor differential conditioning, using 1-min training trials and scoring individual larvae across the complete testing period including the early phases of orientation and presumably indecisiveness, Neuser et al. (2005) found no evidence for fructose memory after one or two training trials, whereas for three, four, and eight training trials these scores were uniformly positive. In the present paradigm, using 2.5-min training trials and end-point counting at the end of the 3-min testing period, our results reveal that even one training trial can be enough to establish fructose memory (Figs. 1A, 4). However, memory scores were higher if two or three training trials were used rather than only one training trial (Fig. 5A), confirming the higher memory scores for the higher number of training trials reported in Neuser et al. (2005). This is also consistent both with common sense and widely accepted prediction-error learning theories (Rescorla and Wagner 1972). Regardless of the number of training trials, memory was not behaviorally expressed in the presence of fructose (Fig. 5B), confirming the data from Figure 4 as well as previous reports (Gerber and Hendel 2006; Schleyer et al. 2011, 2015a). 
A

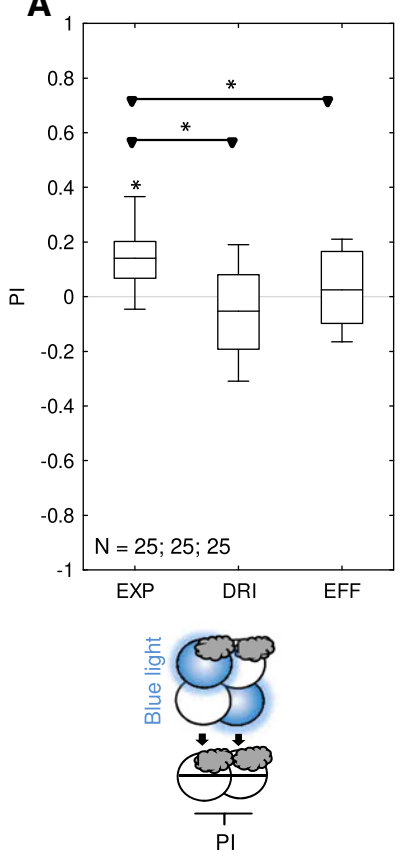

B

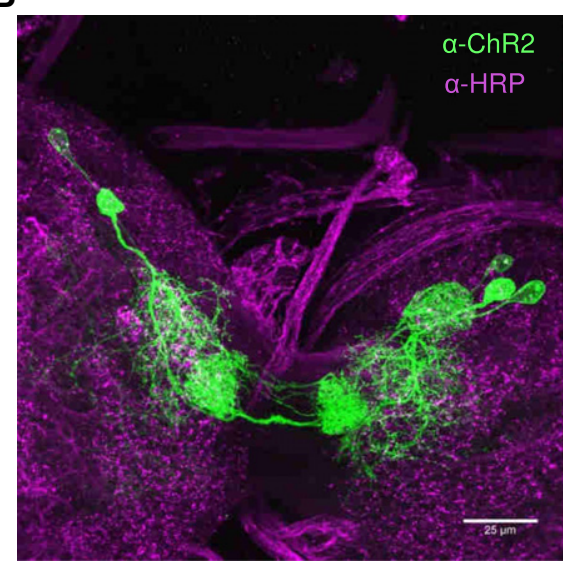

C

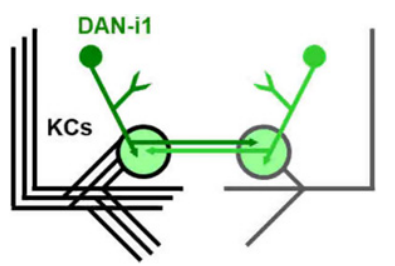

Figure 3. One-trial memory for the association of odor and optogenetic activation of 864-DAN. (A) Larvae were trained by presenting odor and blue light for the optogenetic activation of 864-DAN either together (Paired) or separately (Unpaired), with $2.5 \mathrm{~min}$ trial duration. Differences in odor preference after such training are quantified by the PI and reflect associative memory. The sketches toward the bottom of the figure depict training and testing procedures. Radiating blue Petri dishes indicate light activation during training; Petri dishes with only the substrate, that is pure agarose, but without any tastant added were used throughout. The gray cloud indicates the odor $n$-amylacetate. Pls are positive for the experimental genotype (EXP: 864-DAN>UAS-ChR2-XXL) but not for the genetic controls (DRI: 864-DAN>W1118; EFF: attP40/attP2>UAS-ChR2-XXL), implying that optogenetic activation of 864-DAN leads to reward memory after one training trial. Further details as in Figure 1. Preference scores underlying the PIs are documented in Supplemental Figure S3. (B) Immunohistochemical preparation of the mushroom body region of the experimental genotype. ChR2-XXL is visualized by an anti-ChR2 mouse primary antibody and a Cy3 donkey anti-mouse secondary antibody (green). Confirming an earlier report (Saumweber et al. 2018), this reveals strong and reliable expression in the DAN-i1 neuron of both hemispheres, plus a few additional cell bodies that vary across specimens; Alexa 488 anti-HRP staining yields staining of neuronal membranes for reference (magenta). Data were acquired with a $63 \times$ glycerol objective. (C) Schematic drawing of the left and right mushroom body and the innervation of the upper toe of the medial lobe of the mushroom body by the DAN-i1 neurons of both hemispheres (shown in green).

Notably, the data from Figures 4 and 5 allow an interesting reanalysis. As specified in Equation (2) of the Materials and Methods section, the associative memory scores (i.e., the PI scores) reflect the difference in odor preference between paired-trained and unpaired-trained larvae. The PI scores therefore do not allow a conclusion to be drawn as to whether the animals in the paired group have learned that reward can be found where the odor is, or whether the animals in the unpaired group have learned that reward can be found precisely where the odor is not, or whether both pairedmemory and unpaired-memory are established (see Schleyer et al. 2018 for discussion). This can be revealed, however, by separately considering the Preference scores after paired or unpaired training relative to the "baseline" Preference scores that are observed when the animals are tested in the presence of fructose (Saumweber et al. 2011). Under such test conditions the olfactory behavior of the larvae is cleared of any influence of associative memory (Figs. 4, 5B). Analysis of the Preference scores using this baseline approach reveals surprisingly weak yet statistically significant paired-memory, and relatively robust unpaired-memory (Fig. 6A, combining the data underlying Figs. 4, 5; Supplemental Fig. S6, total sample size of $N=120$ ). The same is observed after three training trials, although in this case paired memory appears more robust (Fig. 6B, $N=40$ ) (also see Schleyer et al. 2018).

\section{Locomotor "footprint" of memory after one-trial training}

Given the above analyses of the Preference scores, we sought to establish the precise mechanisms that produce these results. Larvae navigate odor gradients by a series of relatively straight runs, interrupted by lateral head movements (head casts, HC) that may be followed by turning maneuvers. Appetitive memories after three-trial fructose training have been shown to modulate two aspects of this behavior: the $\mathrm{HC}$ rate and the $\mathrm{HC}$ direction (Schleyer et al. 2015b; Paisios et al. 2017). To see whether the same is the case after one-trial training, we recorded and tracked the animals' behavior in a subset of the experiments shown in Figure $6 \mathrm{~A}$. We found that after paired training the $\mathrm{HC}$ rate-modulation score is higher than after unpaired training, meaning that after paired training the larvae more strongly increase their $\mathrm{HC}$ rate while heading away from the odor source and more strongly decrease their $\mathrm{HC}$ rate while heading toward it (Fig. 6C). Furthermore, the larvae bias their HC direction more toward the odor source after paired training than after unpaired training, indicated by higher reorientation values (Fig. 6D). Interestingly, relative to baseline the only case of significance is for the HC rate-modulation after unpaired training (Fig. 6C,D), which is in line with the robust learning effects in the unpaired-trained animals according to the more "macroscopic" analyses in terms of Preference scores (Fig. 6A).

\section{Fructose memory after one-trial training decays over a few minutes after training}

To study the temporal stability of memory after one-trial training with fructose as the reward, larvae were trained and then tested either immediately after training ( 0 min retention interval), or after retention intervals of respectively 5,10 , or $15 \mathrm{~min}$, which they spent in a water droplet on an otherwise empty Petri dish lid. Appetitive memory was evident immediately after training, whereas results at all later test time-points did not reach significance. Indeed, relative to memory scores immediately after training, scores were decreased when assessed at 5,10 , and 15 min retention intervals (Fig. 7A). Thus, fructose memory after one-trial training is transient, lasting for less than $5 \mathrm{~min}$. For three-trial differential conditioning, using multiple short trials with brief breaks between them, fructose memory was reported to be stable for at least $30 \mathrm{~min}$ in Neuser et al. (2005). For three-trial, single-odor conditioning, memory scores have been reported to be stable for about $20 \mathrm{~min}$ (Kleber et al. 2015). However, under the present conditions, fructose memory after three-trial training was more transient (Fig. 


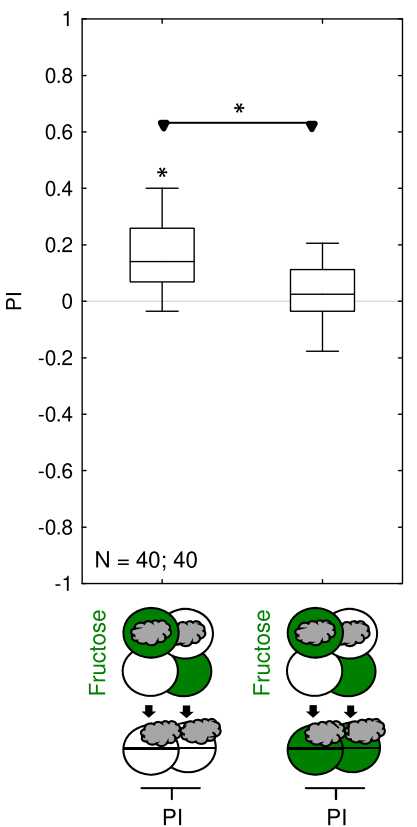

Figure 4. One-trial associative memory is not behaviorally expressed in the presence of fructose during the test. Larvae underwent single training trials of 2.5 min duration, with odor and fructose reward either together (Paired) or separate (Unpaired). Differences in odor preference after such training are quantified by the $\mathrm{PI}$ and reflect associative memory. The sketches toward the bottom of the figures depict the training and testing procedures. Green Petri dishes indicate fructose; dishes without fill indicate Petri dishes with only the substrate, that is pure agarose, but without any tastant added. The gray cloud indicates the odor $n$-amylacetate. Larvae were tested either on pure agarose substrate or in the presence of fructose. If tested on the pure agarose substrate they behaviorally expressed appetitive associative memory (left), whereas no behavioral memory expression was observed in the presence of fructose (right). This is arguably because appetitive associative memory for the odor is expressed in search for the reward, which ceases if the sought-for reward is present. Further details as described in Figure 1. Preference scores underlying the PIs are documented in Supplemental Figure S4.

7B). We note that in this experiment too, the initial memory scores after three training trials were higher than after one training trial (Fig. 7A,B), replicating the results from Figure 5 .

\section{One-trial differential conditioning?}

We next tested whether one-trial memory can also be observed upon differential conditioning. Using fructose as the reinforcer, the larvae either received $n$-amylacetate with reward and 1-octanol without reward (AM+/OCT), or were trained reciprocally (AM/OCT $+)$. Then, we measured the choice between AM and OCT and analyzed the data, with due adjustments, according to equations (1) and (2). This revealed appetitive memory after such one-trial differential conditioning; again, the behavioral expression of this memory was prevented by testing the larvae in the presence of the fructose reward (Fig. 8A). In parallel, we also performed our standard, one-trial learning experiments in the single-odor, absolute conditioning paradigm, using either only AM or only OCT as the odor. Confirming our data from Figures 4 and 5, the use of AM as the odor yielded appetitive memory, the behavioral expression of which was abolished when testing was carried out in the presence of the fructose reward (Fig. 8B). Surprisingly, however, the same type of experiment did not yield evidence for associative memory when using OCT as the odor (Fig. 8C). We thus further investigated whether during differential training any memory accrues to OCT, and whether after training with OCT alone a memory for OCT can be revealed by differentially testing the larvae in a choice situation between AM and OCT.

\section{Do larvae learn about octanol?}

We trained the larvae differentially with fructose as the reward and $\mathrm{AM}$ and OCT as odors, as in Figure 8A; however, we tested the animals for their preference for OCT alone. This revealed that after differential training the larvae did indeed show an OCT memory (Fig. 9A). Moreover, training with OCT alone but differentially testing the larvae for their choice between $\mathrm{AM}$ and OCT also resulted in appetitive memory scores (Fig. 9A). This means that OCT-memory after one-trial training can be revealed if either training or testing is carried out in a differential manner. But is either differential training or differential testing indeed necessary for OCT memory to become detectable? The answer is no, because when three training trials were performed with only OCT during both training and testing the larvae did reveal OCT memory (Fig. 9B) (see also Saumweber et al. 2011; Mishra et al. 2010), a memory which, similar to what we observed for AM in Figure 5, was not behaviorally expressed in the presence of the fructose reward (Fig. 9C).
A

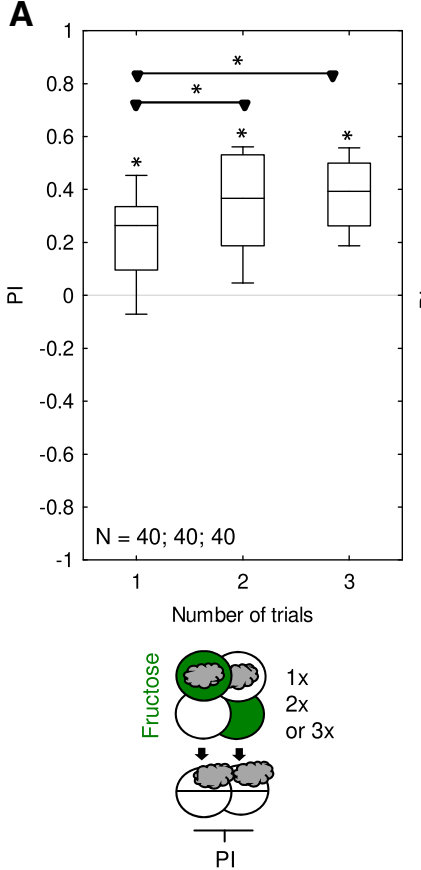

B

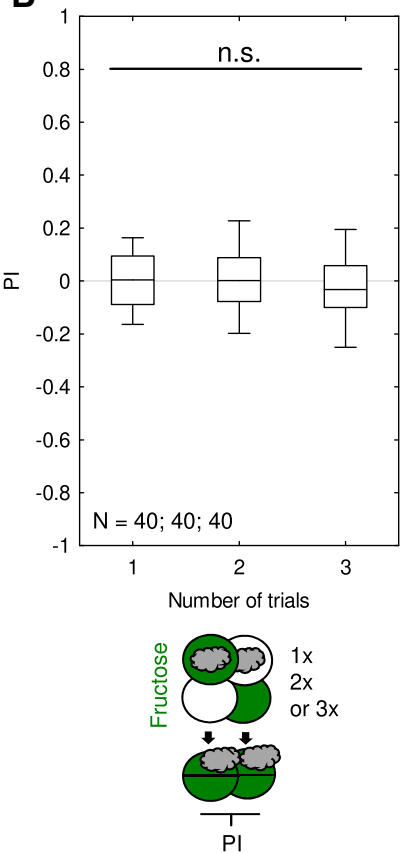

Figure 5. Comparison of associative memory after one, two, and three training trials. Larvae underwent training trials of 2.5 min duration, with odor and fructose reward either together (Paired) or separate (Unpaired). Differences in odor preference after such training are quantified by the PI and reflect associative memory. The sketches toward the bottom of the figures depict the training and testing procedures. Green Petri dishes indicate fructose; dishes without fill indicate Petri dishes with only the substrate, that is pure agarose, but without any tastant added. The gray cloud indicates the odor $n$-amylacetate. Animals were trained either once, twice, or three times. ( $A$ ) If tested on a pure agarose substrate, the larvae showed appetitive memory after one, two, and three training trials; memory scores increased with trial number. $(B)$ If tested in the presence of fructose, appetitive associative memory is not expressed, regardless of the number of training trials. Further details as described in Figure 1. Preference scores underlying the Pls are documented in Supplemental Figure S5. 
A

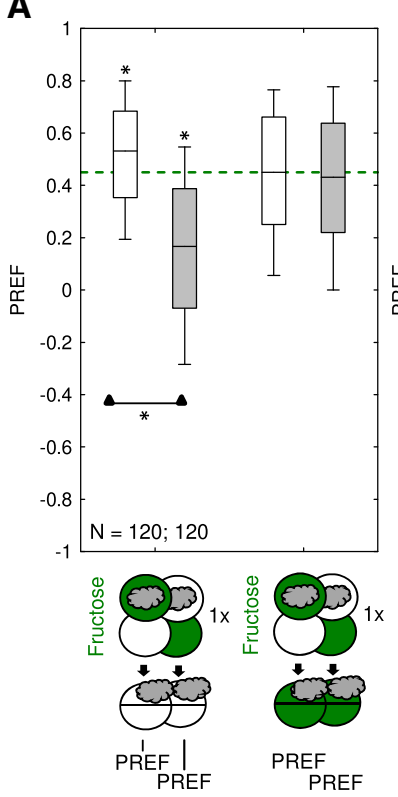

B

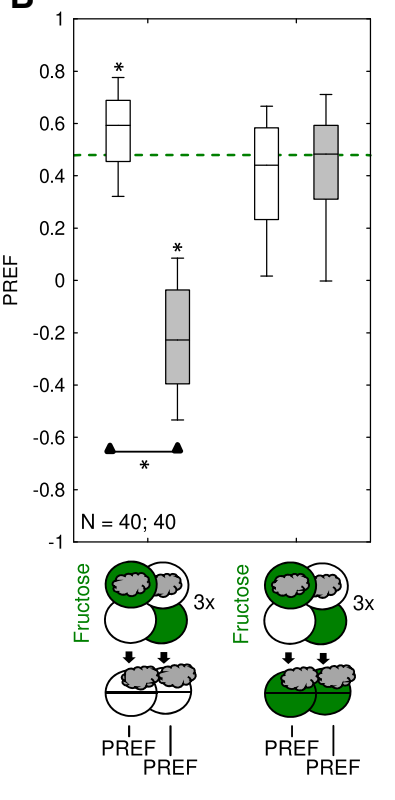

$0 S_{=}^{c}$

D

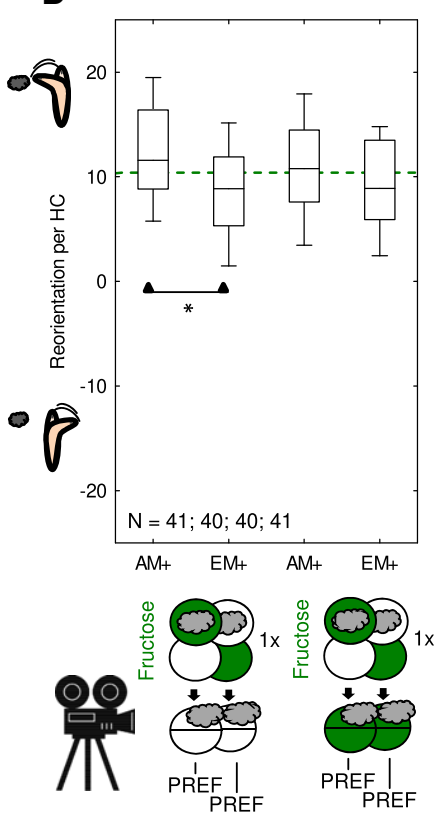

Figure 6. Post-hoc analyses of Preference scores (PREF) and the modulations of locomotion underlying them, after paired and unpaired training. The sketches toward the bottom of the figures depict the training and testing procedures. Green Petri dishes indicate fructose; dishes without fill indicate Petri dishes with only the substrate, that is pure agarose, but without any tastant added. The gray cloud indicates the odor $n$-amylacetate. Further details as described in Figure 1. (A) Analysis of the PREF scores underlying the associative Performance indices (PI) combined from Figures 4 and 5 and Supplemental Figure S6. If tested in the presence of fructose, PREF scores are equal after paired and unpaired training, so their data were pooled, and the median of the pooled data is displayed as the green stippled line indicating baseline odor preference after training, but cleared of associative memory. Relative to this baseline, PREF scores are slightly yet significantly increased for paired training, and are robustly decreased after one-trial unpaired training. (B) Analysis of the PREF scores underlying the associative Performance indices (PI) from Figure 5, after three-trial training. This reveals paired and unpaired memory relative to baseline. Further details as described in Figure 1. $(C, D)$ For a subset of the cases in $(A)$, larvae were video-tracked for offline analyses of their locomotion. This reveals that the behavior of paired-trained and unpaired-trained animals differed quantitatively in terms of the modulation of head cast $(\mathrm{HC})$ rate $(\mathrm{C})$ as well as $\mathrm{HC}$ direction relative to the odor source $(D)$. Relative to baseline, only the modulation of $\mathrm{HC}$ rate after unpaired training was significantly different. Corresponding $\mathrm{P}$ and PREF scores can be found in Supplemental Figure S6. Sketches of larvae $(C, D)$ depict their change in behavior with respect to the odor in the case of positive or negative scores (image courtesy of Naoko Toshima, LIN).

\section{Discussion}

One-trial memory for some, but not all rewards, and for some, but not all punishments

The present analysis reveals that larvae acquire short-term one-trial associative memory of about equal strength for all sugar types tested (Fig. 1E). Thus, it does not seem to be of major importance in this type of assay whether the sugars are both sweet and nutritious (fructose), only sweet (arabinose), or only nutritious (sorbitol) (Fujita and Tanimura 2011; Rohwedder et al. 2012), or indeed whether they promote feeding (Teiichi Tanimura, Nagoya University, Japan, pers. comm.). In adult flies, one-trial short-term memory has likewise been found to be substantial for both nutritious (fructose, glucose, sucrose) and nonnutritious sugars (arabinose, xylose), but only the nutritious sugars supported appetitive memory persisting for at least 24 h (Burke and Waddell 2011). In the larva and for the tested case of fructose, there is no evidence of such longer-term appetitive memory (Fig. 7; Neuser et al. 2005; Kleber et al. 2015). No significant appetitive memory scores were observed in the larva for aspartic acid, a proteinogenic and according to Sang and King (1961) nonessential amino acid (Fig. 1E). Resembling the case of fructose rather than aspartic acid, blue-light activation of the cells covered by the 864-DAN driver strain reveals robust appetitive memory scores after one training trial (Fig. 3).

As regards taste punishment, onetrial aversive associative memory was shown for high-concentration salt (Fig. 2C), matching the report by Widmann et al. (2016), who used a two-odor, differential conditioning paradigm. In contrast, quinine did not elicit significant aversive memory scores (Fig. 2C). We note that, unlike quinine, highconcentration salt may not only affect the taste system of the larvae but also threaten their osmotic balance. Furthermore, exposure of micro-wounds to high-concentration salt may produce itching sensations, potentially in synergy with the activation of multimodal pain sensory neurons, effects that again would not need to be taken into account for quinine. Thus, in the aversive domain, onetrial associative memory might be restricted to very strong kinds of punishment that threaten the larvae's bodily integrity. This might not be the case for brief exposures to high-concentration salt (Fig. 2C). We note that a trend rather for appetitive memory through such brief 
A

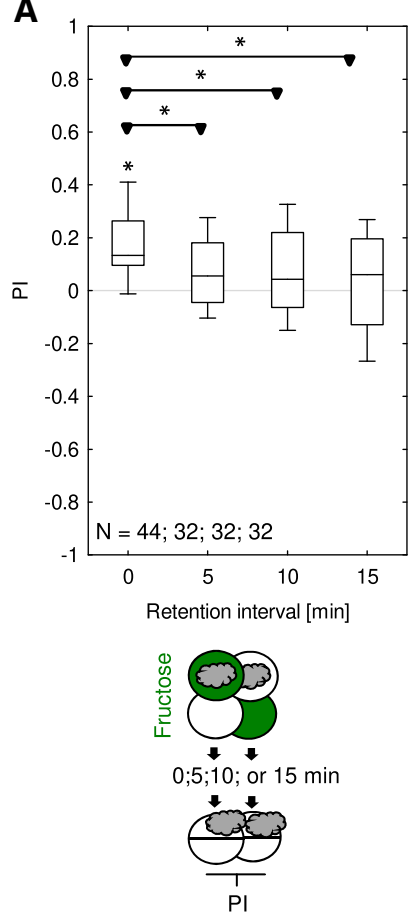

B

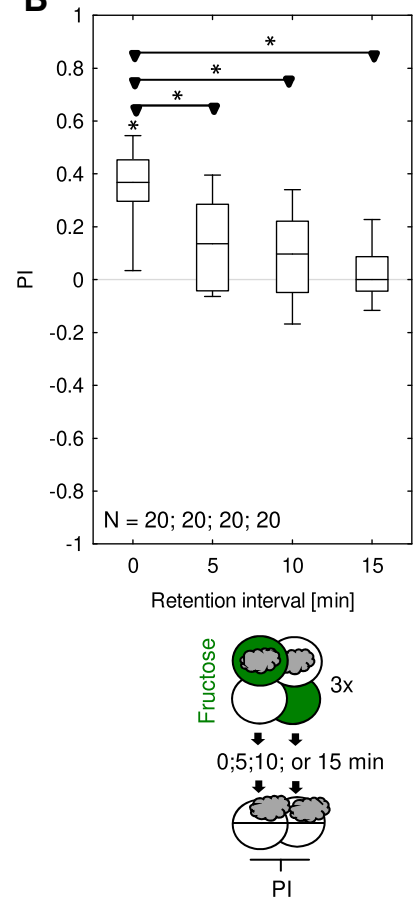

Figure 7. Temporal dynamics of one-trial associative memory using fructose as reward. Larvae underwent training trials of 2.5 min duration, with odor and fructose reward either together (Paired) or separate (Unpaired). Differences in odor preference after such training are quantified by the $\mathrm{PI}$ and reflect associative memory. Larvae were tested either immediately after training (retention interval $0 \mathrm{~min}$ ), or 5,10 , or $15 \mathrm{~min}$ after training. The sketches toward the bottom of the figures depict training and testing procedures. Green Petri dishes indicate fructose; dishes without fill indicate Petri dishes with only the substrate, that is pure agarose, but without any tastant added. The gray cloud indicates the odor $n$-amylacetate. Regardless of whether training was performed with $(A)$ only one trial or $(B)$ three trials, memory scores were significant for immediate testing whereas results at the later time-points remained below the statistical threshold. Further details as described in Figure 1. Preference scores underlying the Pls are documented in Supplemental Figure S7.

exposure (Fig. 2A,C) would be consistent with the report by Gerber and Hendel (2006), who showed that low-concentration salt, which might likewise not threaten bodily integrity, has a rewarding effect in larvae (Gerber and Hendel 2006).

\section{Practical implications}

The present one-trial version of the paradigm can substitute for the lengthier three-trial version, at least for those reinforcers that indeed support one-trial memory. It is also more resource-friendly, requiring fewer Petri dishes, less agarose, and lower quantities of tastant substances, making it environmentally and financially preferable over the three-trial version. For teaching purposes in high school classroom settings or for undergraduate laboratory courses (Michels et al. 2017), one-trial training with a training trial duration of $2.5 \mathrm{~min}$ and using single-odor absolute conditioning with fructose as the reward might therefore be the procedure of choice.

\section{Sources of variability}

Variability in odor-fructose associative memory scores, and in the preference scores underlying them, is apparently higher after one-

trial training than after three-trial training (Figs. 5A, 6A,B). Is this because the effect of odor exposure, which decreases odor preference (larvae: Michels et al. 2005; Saumweber et al. 2018, for discussion of earlier work see Gerber and Stocker 2007; adults: Pech et al. 2015; Hattori et al. 2017), and the effect of reward exposure, which increases odor preference compared to innate odor preference (larvae: Michels et al. 2005; Saumweber et al. 2011; Saumweber et al. 2018; adults: not reported), are greater on one-trial memory scores than on three-trial memory scores? Indeed, one-trial associative memory might be only partially consolidated at the moment of testing, allowing the two aforementioned, exposure-induced processes to influence behavior more strongly. This might make behavior more variable because, as noted above, these processes are of opposite effect on odor preference. In contrast, after repeated training the consolidation of associative memories might already be complete, at least for the early trials, and might dominate behavior.

A second source of variability after one- rather than three-trial training might be related to our current finding that memory after one training trial is apparently dominated by the effects of unpaired rather than paired training (Fig. 6). Why would this lead to particularly variable results when only one training trial is used? Within the framework of models of associative learning using prediction-error learning rules (Rescorla and Wagner 1972), one might suggest that the presentation of fructose in the absence of odor establishes context-fructose memory. If subsequently the odor is presented within that same context, a prediction error arises: fructose is predicted by contextual cues, but it is not actually received. This would be the basis for the change in valence of the odor presented during this "frustrating" experience (for review, see Schleyer et al. 2018). Obviously, according to such a scenario the presentation of fructose has to come before the presentation of the odor, as is the case for half of the repetitions within each of our experiments. If the order of presentation is reversed-as is the case in the other half of our samples-the context would still be neutral at the moment of odor presentation. As training progresses with subsequent trials, this variation in the effectiveness of one-trial unpaired training would eventually be diluted out.

Thus, behavior after only one training trial is arguably affected more strongly than after three training trials by the effects of odor exposure and of fructose exposure, and by variations in the effectiveness of learning through unpaired training. If the target associative memory component is weak to begin with, as in the case of aspartic acid and quinine, this may render one-trial associative memory practically undetectable (Figs. 1D, 2B).

\section{The role of differential training and testing for learning about 1-octanol}

We found that one-trial associative memory for 1-octanol requires either differential training or differential testing (Figs. 8C, 9A). The requirement for differential training points to a plasticity mechanism during training as a prerequisite for a memory trace for 1-octanol even to be established. This is reminiscent of what Mishra et al. (2010) reported for the larva, and Barth et al. (2014) as well as König et al. (2017) for adult Drosophila, in all cases using 3-octanol and 1-octen-3-ol as the odor pair. These authors noted that after absolute, single-odor training the animals fully generalize between these two odors, that is they behave toward the nontrained odor in the same way as toward the trained odor. It is only after differential training that behavior is selective for the trained odor. Barth et al. (2014) further showed that such acuity learning involves both second- and third-order olfactory processing stages.

The requirement for differential testing to reveal one-trial memory for 1-octanol in the present study implies that during 
A

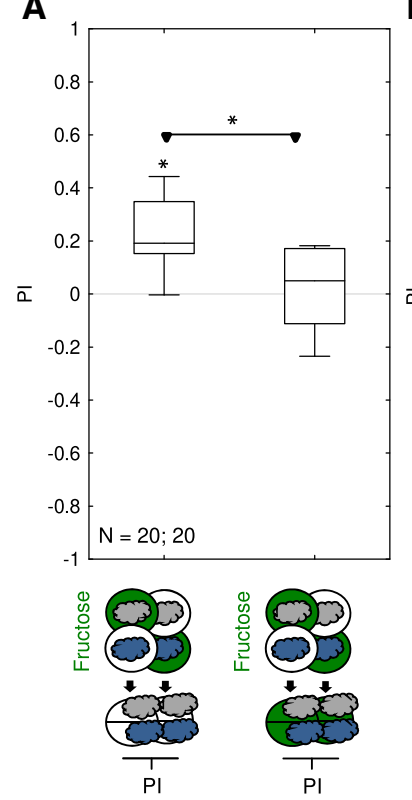

B

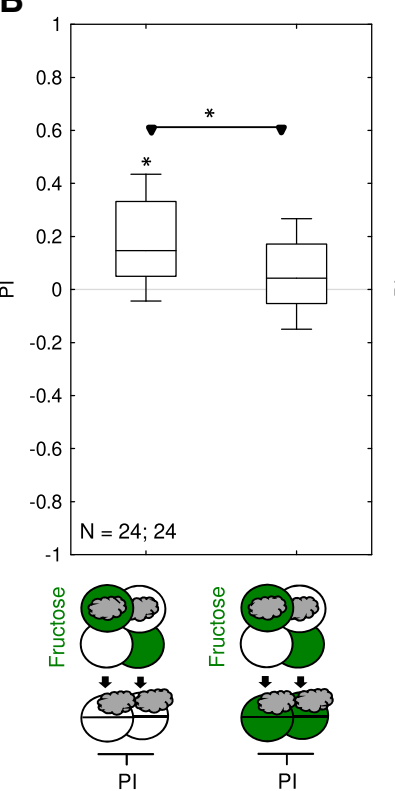

C

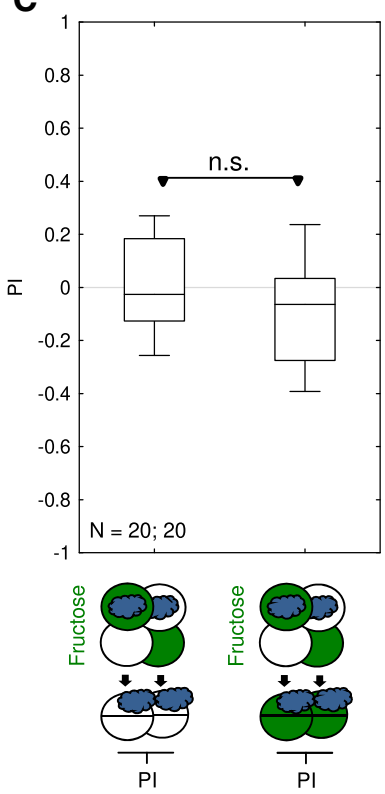

Figure 8. One-trial associative memory for $n$-amylacetate but not for 1-octanol? Larvae underwent single training trials of 2.5 min duration, with odors and fructose reward at the contingencies indicated. Differences in odor preference after such training are quantified by the PI and reflect associative memory. The sketches toward the bottom of the figures depict training and testing procedures. Green Petri dishes indicate fructose; dishes without fill indicate Petri dishes with only the substrate, that is pure agarose, but without any tastant added. The gray cloud indicates the odor $n$-amylacetate, and the dark blue cloud the odor 1-octanol. $(A)$ Animals were trained differentially, with one of the two odors $n$-amylacetate or 1-octanol paired with fructose and the other odor presented alone. Then, the relative preference between the two odors was determined in a choice test and PIs were calculated. The data show appetitive associative memory when testing was carried out on pure agarose Petri dishes, whereas the behavioral expression of memory was abolished when testing was carried out in the presence of fructose. $(B)$ As in $(A)$, but omitting 1 -octanol. Appetitive memory for $n$-amylacetate is behaviorally expressed in the absence but not in the presence of fructose (also see Figs. 4, 5). (C) As in $(A)$, but omitting $n$-amylacetate. Regardless of the test condition, no associative memory for 1 -octanol is detectable. Further details as described in Figure 1. Preference scores underlying the Pls are documented in Supplemental Figure S8.

testing 1-octanol is processed more effectively for memory retrieval when presented in the context of $n$-amylacetate as a choice alternative than when presented alone. During the test the presence of a second odor gradient, oriented at $180^{\circ}$ to the "target" odor, possibly helps the animals to navigate toward their target.

\section{Stronger, or different, memories after one- versus three-trial training?}

Odor-fructose associative memory scores increase across training trials (Fig. 5A; see also Neuser et al. 2005). Does this come about by an increase in strength of the association, or by the recruitment of a different, additive kind of process in the trials following the first one? Although the former seems to be the more parsimonious explanation, results from the honeybee suggest that one- and threetrial training establish memories that differ in kind rather than in strength alone. In appetitive classical conditioning of the proboscis extension reflex (PER), one-trial memory thus begins to decay after about one day, whereas three or more conditioning trials induce memory that is stable for up to several days (for review, see Giurfa and Sandoz 2012). Critically, unlike one-trial memory, memory after three training trials in this paradigm is largely amnesia-resistant and dependent on translation and transcription (for reviews, see Schwärzel and Müller 2006; but see Pamir et al. 2014). It is interesting to note that memories established by one-trial learning might in themselves be composite, as has been shown by Scheunemann et al. (2013) by varying the number and intensity of individual shock pulses within single training trials in wild-type versus mutant adult Drosophila.

We note that an interpretation of acquisition fundamentally different from the above was offered by Pamir et al. (2014). On the basis of the individual-animal performance of honeybees during and after one-trial or multiple-trial PER conditioning, the authors argue that in individual animals learning is actually a step-like process (also see Trabasso 1963 and Ohl et al. 2001 for examples of such one-trial learning during category formation in humans and gerbils, respectively). Once the animals have started to show learned behavior in the second trial, for example, they continue to do so during further acquisition trials. What increases across training trials, the authors argue, is the frequency of individual bees that turn into responders. Thus, the gradual appearance of an "acquisition curve" is suggested to be an artifact of averaging across animals. Indeed, 24-h-memory was similarly strong regardless of whether individual bees started to show learned behavior after the first or after subsequent training trials.

\section{“Small data" learning}

In biological systems, one-trial associative learning is not unusual (see opening section). It should be of evolutionary benefit whenever, despite the limited predictive evidence that only one training trial can offer, the cost of wrongly not-predicting the outcome is higher than the cost of wrongly predicting it. Under such conditions, biological systems are apparently capable of lean, "small data" predictive learning that contrasts with the powerful and heavily energy-consuming "big data" predictive strategies of artificial intelligence (Halevy et al. 2009; Obermeyer and Emanuel 2016).

\section{Materials and Methods}

This study uses established methods for odor-taste associative learning in larval Drosophila (Scherer et al. 2003; Gerber and Hendel 2006; El-Keredy et al. 2012; Schleyer et al. 2015a), unless mentioned otherwise using the single-odor, absolute conditioning paradigm established for fructose as tastant by Saumweber et al. (2011). In principle, one group of larvae receives an odor together with a tastant reinforcer (paired), whereas a second group is presented with the odor and the tastant separately from each other (unpaired). After such training, both groups are tested for their preference for the odor. Differences in odor preferences between paired-trained and unpaired-trained groups thus indicate associative memory. A distinct feature of the present study is that we use only one training trial, unless mentioned otherwise.

\section{Animals}

We used 5-d-old, third instar, feeding-stage larvae from the Canton-S wild-type strain. Flies were maintained at $25^{\circ} \mathrm{C}, 60 \%-$ 
A

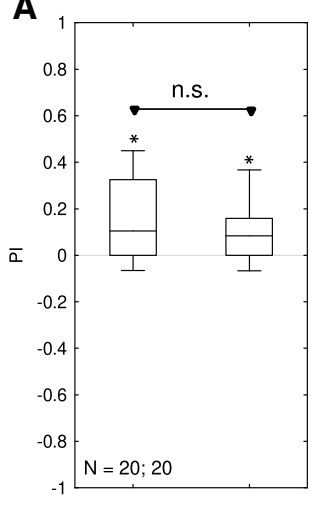

B

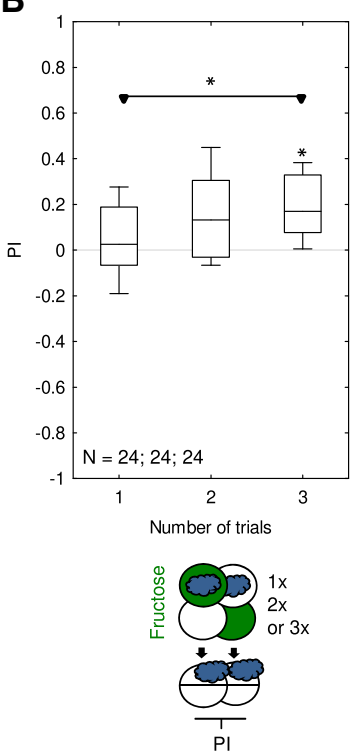

C

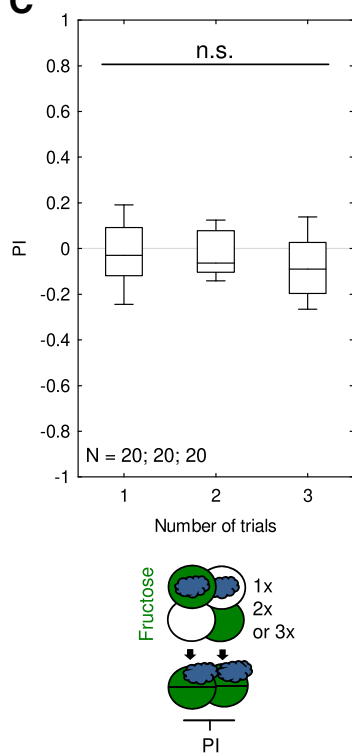

Figure 9. One-trial associative memory for 1-octanol is revealed by differential training or differential testing; associative memory is also revealed by using multiple training trials. Larvae underwent training trials of 2.5 min duration, with odors and fructose reward at the number and contingencies indicated. Differences in odor preference after such training are quantified by the $\mathrm{PI}$ and reflect associative memory. The sketches toward the bottom of the figures depict training and testing procedures. Green Petri dishes indicate fructose; dishes without fill indicate Petri dishes with only the substrate, that is pure agarose, but without any tastant added. The gray cloud indicates the odor $n$-amylacetate, and the dark blue cloud the odor 1-octanol. (A) (Left) Animals were trained differentially, with one of the two odors $n$-amylacetate or 1 -octanol paired with fructose and the other odor presented alone. Then, the preference for 1-octanol was determined and PIs were calculated, revealing associative memory for 1-octanol. (A) (Right) Animals were trained with 1-octanol either paired or unpaired with the fructose reward. Then, they were tested for their choice between 1-octanol and $n$-amylacetate, revealing that 1-octanol training has established associative memory. $(B)$ Animals received either paired or unpaired training with 1-octanol and fructose reward, either once, twice, or three times, and were then tested for their 1-octanol preference. This reveals associative memory for 1 -octanol after three but no fewer training trials. (C) As in $(B)$ but testing the larvae in the presence of fructose; under these conditions, the behavioral expression of associative memory for 1-octanol was abolished. Further details as described in Figure 1. Preference scores underlying the PIs are documented in Supplemental Figure S9.

$70 \%$ relative humidity and a $12 / 12 \mathrm{~h}$ light-dark cycle. Cohorts of approximately 30 larvae were collected from the food vials, rinsed in water, collected in a water droplet and subsequently used in the respective experiment.

Furthermore, we used transgenically modified larvae to optogenetically activate a specific set of neurons. We crossed animals of the effector strain UAS-ChR2-XXL (Bloomington Stock Center no. 58374; Dawydow et al. 2014) to animals of the split-Gal4 driver strain SS00864-Gal4 to obtain double-heterozygous offspring. In the larvae in question, the blue-light-gated cation channel ChR2-XXL can be activated in the neurons of interest. The expression pattern of the SS00864-Gal4 driver strain was confirmed by immunohistochemistry to include the hemispherically unique DAN-i1 neurons, with additional stochastic expression in 1-2 further mushroom body extrinsic neurons (Saumweber et al. 2018); it is henceforth called 864-DAN (see Immunohistochemistry section). As the driver control, we crossed the 864-DAN driver strain to our local copy of $\mathrm{w}^{1118}$ (Bloomington Stock Center no. 3605, $5905,6326)$. As the effector control, a strain homozygous for both landing sites used for the split-Gal4 (attP40/attP2), yet without a Gal4 domain inserted, was crossed to UAS-ChR2-XXL (Pfeiffer et al. 2010). All transgenic flies were raised in darkness with black cardboard wrapped around the food vial.

\section{Odor-fructose associative learning}

For paired training, cohorts of 30 larvae were placed at the center of a Petri dish ( $9 \mathrm{~cm}$ inner diameter; Sarstedt) equipped with odor containers (see below) and filled with $1 \%$ agarose solution (electrophoresis grade; Roth) supplemented with fructose (FRU; 2 M; CAS: $57-48-7$, purity 99\%, Roth) as a taste reward (+). Custommade Teflon containers of $5 \mathrm{~mm}$ diameter contained $10 \mu \mathrm{L}$ of odor substance. This was either $n$-amylacetate (AM; CAS: 628-63-7, Merck), diluted 1:20 in paraffin oil (CAS: 8042-47-5, AppliChem), or 1-octanol (OCT; CAS: 111-87-5, Merck), undiluted. Paraffin has no behavioral significance as an odor (Saumweber et al. 2011). The containers were closed by a lid perforated with 5-10 holes, each of $\sim 0.5 \mathrm{~mm}$ diameter. At each of the opposing edges of the Petri dish (left or right), one odor container holding AM was placed. Larvae were then free to move about this AM+ Petri dish for $2.5 \mathrm{~min}$, unless mentioned otherwise. Then, they were transferred to a Petri dish which lacked fructose and which featured two empty odor containers (EM), and they were left there for the same amount of time. After such AM+/EM training, they were transferred to the center of a test Petri dish, where an AM odor container was presented on one side and an empty odor container on the opposite side, and were thus tested for their preference for AM. Unless mentioned otherwise, the test Petri dish featured only agarose, but no added tastant. After $3 \mathrm{~min}$, the number of larvae (\#) on the AM side, on the EM side, and in a $10-\mathrm{mm}$ wide middle zone was counted. Larvae crawling up the side-walls of the Petri dish were counted for the respective side, whereas larvae on the lid were excluded from the analysis. A preference index (PREF) was calculated:

$$
\mathrm{PREF}=\frac{(\# \mathrm{AM}-\# \mathrm{EM})}{\# \text { Total }} .
$$

Preference indices may thus range from +1 to -1 , with positive values indicating preference and negative values indicating avoidance of AM. Across repetitions of the experiments, in half of the cases the sequence was as indicated $(\mathrm{AM}+/ \mathrm{EM})$, whereas in the other cases it was reversed (EM/AM+).

The procedure for unpaired training was the same, except that the Petri dishes featured either only AM or only the reward. After such $\mathrm{AM} / \mathrm{EM}+$ training (again in half of the cases the sequence was reversed: $\mathrm{EM}+/ \mathrm{AM}$ ), the preference test was carried out as above.

From the PREF scores after paired and unpaired training, a PI was calculated:

$$
\mathrm{PI}=\frac{(\text { PREF Paired }- \text { PREF Unpaired })}{2}
$$

Thus, performance indices may range from +1 to -1 . Positive PIs indicate appetitive associative memory, whereas negative values indicate aversive associative memory.

\section{Odor-arabinose associative learning}

The procedure was as described above for fructose, except that instead of fructose we used arabinose as a reward (ARA; 2 M; CAS: 10323, purity $\geq 98 \%$, Sigma-Aldrich). 
Odor-sorbitol associative learning

The procedure was as described above for fructose, except that instead of fructose we used sorbitol as a reward (SOR; 2 M; Art-Nr. 6212.2 , purity $\geq 98 \%$, Roth).

\section{Odor-aspartic acid associative learning}

The procedure was as described above for fructose, except that instead of fructose we used aspartic acid as a reward (ASP; $10 \mathrm{mM}$; CAS: $56-84-8$, purity $\geq 99 \%$, Sigma-Aldrich).

\section{Odor-quinine associative learning}

The procedure was as described above for fructose, with two exceptions. First, instead of fructose we used quinine hemisulfate as punishment (QUI; 5 mM; CAS: 6119-70-6, purity 92\%, Sigma-Aldrich). Second, QUI was present during testing. This is because learned avoidance of quinine-associated odors is a form of escape behavior that is expressed only if the test situation warrants escape, that is if it includes the quinine punishment to motivate escape (Gerber and Hendel 2006; Schleyer et al. 2011, 2015a).

\section{Odor-sodium chloride associative learning}

The procedure was as described above for quinine, except that instead of quinine we used a high sodium chloride concentration as a punishment (high salt/NaCl; $1.5 \mathrm{M}$; Art-Nr. 3957.1, purity $\geq 99.5 \%$, Roth). As with quinine, only when the tests are carried out in the presence of high-concentration salt as the negative reinforcer are the larvae prompted to express aversive memory behaviorally (Gerber and Hendel 2006; Niewalda et al. 2008; Widmann et al. 2016).

\section{Odor-DAN associative learning}

All optogenetic experiments were performed inside a custom-made box. Within the box, a light table was equipped with $24 \times 12$ LEDs with a peak wavelength of $470 \mathrm{~nm}$ (Solarox), with a $6 \mathrm{~mm}$-thick diffusion plate of frosted Plexiglas on top to ensure uniform light conditions and intensity $\left(120 \mu \mathrm{W} / \mathrm{cm}^{2}\right)$. The Petri dishes for the learning assay were placed directly on top of the diffusion plate surrounded by a ring of 30 infrared LEDs ( $850 \mathrm{~nm}$; Solarox) behind a polyethylene diffusion ring that provided illumination. Similarly to the associative learning experiment described above, we trained larvae either paired or unpaired with the reinforcer, which in this case consisted of optogenetic activation of 864-DAN rather than a tastant. Each trial lasted $2.5 \mathrm{~min}$ and the larvae were trained in one training cycle only. Then the larvae were transferred to a pure test Petri dish, and their preference for AM as well as the PI was calculated as detailed above. Experimenters were blind to genotype.

Whenever variations in the above paradigms were used, these are mentioned along with the presentation of the results.

\section{Locomotor footprint of memories established by odor-fructose associative learning}

Larval behavior was video-tracked and analyzed as described in detail in Paisios et al. (2017). In brief, two aspects of larval chemotaxis were analyzed. First, the modulation of head cast $(\mathrm{HC})$ rate:

$$
\mathrm{HC} \text { rate-modulation }=\frac{(\# \mathrm{HC} / \mathrm{s}(\text { heading away })-\# \mathrm{HC} / \mathrm{s}(\text { heading towards }))}{(\# \mathrm{HC} / \mathrm{s}(\text { heading away })+\# \mathrm{HC} / \mathrm{s}(\text { heading towards }))} .
$$

This measure yields positive scores for attraction, that is when larvae systematically perform more head casts while heading away from the odor (i.e., when odor concentration decreases) than while heading toward it (i.e., when odor concentration increases). Conversely, it yields negative scores for aversion.
Second, the modulation of head cast direction was measured by the reorientation per head cast:

Reorientation per $\mathrm{HC}=\mathrm{abs}($ heading angle before $\mathrm{HC}$ ) - abs(heading angle after $\mathrm{HC}$ )

In this measure, the heading angle describes the orientation of the animal's head relative to the odor, with absolute heading angles of $0^{\circ}$ or $180^{\circ}$, for example, indicating that the odor is to the front or to the rear of the larvae, respectively. This measure thus yields positive scores for attraction, that is when the head cast directs the larvae toward rather than away from the odor target, whereas it yields negative scores for aversion.

\section{Immunohistochemistry}

We undertook larval body wall preparations $(N=4)$ (see Budnik et al. 2006) on larvae of the same experimental genotype as used for our optogenetic one-trial experiment, for which the 864-DAN driver strain had been crossed to the UAS-ChR2-XXL effector strain. Larvae were individually placed at the center of a well in a custom-made magnetic chamber (kindly provided by Dr. Ulrich Thomas, LIN). Pinning the anterior and posterior ends, larvae were covered with $\mathrm{Ca}^{2+}$-free saline and then dissected using microscissors (No. 15002-08, Fine Science Tools GmbH). The internal organs were removed, whereas the central nervous system was left intact. After washing again with $\mathrm{Ca}^{2+}$-free saline, the larvae were fixed in Bouin's solution (HT10132, Sigma-Aldrich) for $7 \mathrm{~min}$ and afterwards briefly washed three times in $0.2 \%$ PBT and then another three times every $15 \mathrm{~min}$. The larvae were then transferred into a glass bowl and treated overnight at $4^{\circ} \mathrm{C}$ with the primary monoclonal anti-ChR2 mouse antibody (ProGen Biotechnik) diluted 1:100 in $0.2 \%$ PBT. After three washing steps once every 10 min in $0.2 \%$ PBT, tissue was incubated with a secondary Cy3 donkey anti-mouse (Dianova) and a HRP Alexa Fluor 488 goat antihorseradish peroxidase (Jackson Immuno Research), both diluted 1:300 in $0.2 \%$ PBT for $1 \mathrm{~h}$. After three final washing steps once every 10 min with $0.2 \%$ PBT, samples were mounted in Vectashield (Vector Laboratories Inc.) on a cover slip. Preparations were examined under a DM6000 CS confocal microscope (Leica). All image stacks were analyzed with Image-J (NIH) software.

\section{Statistics}

For the behavioral data, nonparametric statistics were applied throughout. For comparisons with chance levels (i.e., with zero), one-sample sign tests (OSS; corresponding to binom.test) were used (R Development Core Team 2016). For between-group comparisons, Kruskal-Wallis (KW) and Mann-Whitney U-tests (MWU) were applied where appropriate (Statistica 13 from StatSoft). We used a BH correction for multiple comparisons to maintain an error rate below 5\% (Holm 1979). We speak of a trend toward significance when a given comparison would be significant without such correction. Data are displayed as box plots with the median as the middle line, the box boundaries as 25\% and $75 \%$ quantiles and the whiskers as $10 \%$ and $90 \%$ quantiles; they are documented in the Supplemental Data files.

\section{Competing interests statement}

The authors declare no competing interests.

\section{Acknowledgments}

Discussions with Birgit Michels (LIN), Christian König (LIN), Michael Brunk (LIN), Markus Fendt (OVGU Magdeburg), Fabian Bötzl (U Würzburg), and Robert Kittel (U Leipzig) are gratefully acknowledged, as are the technical assistance and experimental contributions of Michael Thane, Stefan Stögbauer, Elisabeth Orlov, Corinna Schwenke, Louisa Warzog, Jan Wöllmann, Celina Dölle, and Niclas Groth as well as the contributions to immunohistochemistry of Oliver Kobler (LIN). We thank Naoko Toshima 
(LIN) for allowing us to use the larval sketches in Figure 6, and R.D. V. Glasgow (Zaragoza, Spain) for language editing. This study received institutional support by the Otto von Guericke Universität Magdeburg (OVGU), the Wissenschaftsgemeinschaft Gottfried Wilhelm Leibniz (WGL), the Leibniz Institute for Neurobiology (LIN), as well as grant support from the Deutsche Forschungsgemeinschaft (DFG) (GE 1091/4-1, CRC 779 Motivated behavior, and FOR 2705 Mushroom body, to B.G.).

Author contributions: A.W., M.S., and B.G. designed the experiments. A.W., F.G., and N.M. performed the experiments. A.W. did the statistical analyses on the data. A.W. and B.G. wrote the manuscript with input from all authors.

\section{References}

Abt JP, Essmann WB, Jarvik ME. 1961. Ether-induced retrograde amnesia for one-trial conditioning in mice. Science 133: 1477-1478. doi:10.1126/ science.133.3463.1477

Apostolopoulou A, Mazija L, Wüst A, Thum AS. 2014. The neuronal and molecular basis of quinine-dependent bitter taste signaling in Drosophila larvae. Front Behav Neurosci 8: 6. doi: 10.3389/fnbeh.2014.00006

Aso Y, Rubin GM. 2016. Dopaminergic neurons write and update memories with cell-type-specific rules. eLife 5: e16135. DOI: 10.7554/eLife.16135

Barth J, Dipt S, Pech U, Hermann M, Riemensperger T, Fiala A. 2014. Differential associative training enhances acuity in Drosophila melanogaster. J Neurosci 34: 1819-1837. doi:10.1523/JNEUROSCI .2598-13.2014

Beck CDO, Schroeder B, Davis RL. 2000. Learning performance of normal and mutant Drosophila after repeated conditioning trials with discrete stimuli. J Neurosci 20: 2944-2953. doi:10.1523/JNEUROSCI 20-08-02944.2000

Blank M, Guerim LD, Cordeiro RF, Vianna MRM. 2009. A one-trial inhibitory avoidance task to zebrafish: rapid acquisition of an NMDA-dependent long-term memory. Neurobiol Lern Mem 92: 529-534. doi:10.1016/j.nlm.2009.07.001

Budnik V, Gorczyca M, Prokop A. 2006. Selected methods for the anatomical study of Drosophila embryonic and larval neuromuscular junctions. Int Rev Neurobiol 75: 323-65. doi:10.1016/S0074-7742(06)75015-2

Burke CJ, Waddell S. 2011. Remembering nutrient quality of sugars in Drosophila. Curr Biol 21: 746-750. doi:10.1016/j.cub.2011.03.032

Cammarota M, Bevilaqua LRM, Köhler C, Medina JH, Izquierdo I. 2005. Learning twice is different from learning once and from learning more. Neuroscience 132: 273-279. doi:10.1016/j.neuroscience.2005.01.022

Cherkin A. 1969. Kinetics of memory consolidation: role of amnesic treatment parameters. Proc Natl Acad Sci 63: 1094-101. doi:10.1073/ pnas.63.4.1094

Colomb J, Kaiser L, Chabaud M-A. 2009. Parametric and genetic analysis of Drosophila appetitive long-term memory and sugar motivation. Genes Brain Behav 4: 407-415. doi:10.1111/j.1601-183X.2009.00482.x

Dawydow A, Gueta R, Ljaschenk D, Ullrich S, Hermann M, Ehmann N, Gao S, Fiala A, Langenhan T, Nagel G, et al. 2014.

Channelrhodopsin-2-XXL, a powerful optogenetic tool for low-light applications. Proc Natl Acad Sci 111: 13972-13977. doi:10.1073/pnas .1408269111

Diegelmann S, Klagges B, Michels B, Schleyer M, Gerber B. 2013. Maggot learning and Synapsin function. I Exp Biol 216: 939-951.

Duffy JB. 2002. GAL4 system in Drosophila: a fly geneticist's Swiss army knife. Genesis 34: 1-15. doi:10.1002/gene.10150

El-Keredy A, Schleyer M, König C, Ekim A, Gerber B. 2012. Behavioural analyses of quinine processing in choice, feeding and learning of larval Drosophila. PLoS One 7: e40525. doi:10.1371/journal.pone.0040525

Eschbach C, Cano C, Haberkern H, Schraut K, Guan C, Triphan T, Gerber B. 2011. Associative learning between odorants and mechanosensory punishment in larval Drosophila. J Exp Biol 214: 3897-3905. doi:10 $.1242 /$ jeb.060533

Fujita M, Tanimura T. 2011. Drosophila evaluates and learns the nutritional value of sugars. Curr Biol 21: 751-755. doi:10.1016/j.cub.2011.03.058

Gerber B, Hendel T. 2006. Outcome expectations drive learned behaviour in larval Drosophila. Proc Biol Sci 273: 2965-2968. doi:10.1098/rspb.2006 3673

Gerber B, Stocker T. 2007. The Drosophila larva as a model for studying chemosensation and chemosensory learning: a review. Chem Senses 32: 65-89. doi:10.1093/chemse/bj1030

Giurfa M, Sandoz J. 2012. Invertebrate learning and memory: fifty years of olfactory conditioning of the proboscis extension response in honeybees. Learn Mem 19: 54-66. doi:10.1101/1m.024711.111

Haesen K, Beckers T, Baeyens F, Vervliet B. 2017. One-trial overshadowing: evidence for fast specific fear learning in humans. Behav Res Ther 90: 1624. doi:10.1016/j.brat.2016.12.001
Halevy A, Norvig P, Pereira F. 2009. The unreasonable effectiveness of data. IEEE Intell Syst 24: 8-12. doi:10.1109/MIS.2009.36

Hattori D, Aso Y, Swartz KJ, Rubin GM, Abbot LF, Axel R. 2017. Representations of novelty and familiarity in a mushroom body compartment. Cell 169: 956-969. doi:10.1016/j.cell.2017.04.028

Hilliard S, Nguyen M, Domjan M. 1997. One-trial appetitive conditioning in the sexual behavior system. Psychon Bull Rev 4: 237-241. doi:10.3758/ BF03209399

Hoffmann J, Berner M, Butz MV, Herbort O, Kiesel A, Kunde W, Lenhard A. 2007. Explorations of anticipatory behavioral control (ABC): a report from the cognitive psychology unit of the University of Würzburg. Cogn Process 8: 133-142. doi:10.1007/s10339-007-0166-y

Holm S. 1979. A simple sequentially rejective multiple test procedure. Scand J Statist 6: $65-70$.

Housden BE, Perrimon N. 2016. Cas9-mediated genome engineering in Drosophila melanogaster. Cold Spring Harb Protoc 2016: pdb top086843. doi:10.1101/pdb.top086843

Kleber J, Chen Y, Michels B, Saumweber T, Schleyer M, Kähne T, Buchner E, Gerber B. 2015. Synapsin is required to 'boost' memory strength for highly salient events. Learn Mem 23: 9-20. doi:10.1101/lm.039685.115

König C, Antwi-Adjei E, Ganesan M, Kilonzo K, Viswanathan V, Durairaja A, Voigt A, Yarali A. 2017. Aversive olfactory memory loses odor specificity over time. J Exp Biol 220: 1548-1553. doi:10.1242/jeb.155317

König C, Khalili A, Ganesan M, Nishu AP, Garza AP, Niewalda T, Gerber B, Aso Y, Yarali A. 2018. Reinforcement signaling of punishment versus relief in fruit flies. Learn Mem 25: 247-257. doi:10.1101/lm.047308.118

Li HH, Kroll JR, Lennox SM, Ogundeyi O, Jeter J, Depasquale G, Truman JW. 2014. A GAL4 driver resource for developmental and behavioral studies on the larval CNS of Drosophila. Cell Rep 8: 897-908. doi:10.1016/j .celrep.2014.06.065

Neuser K, Husse J, Stock P, Gerber B. 2005. Appetitive olfactory learning in Drosophila larvae: testing for effects of training amount, reinforcer intensity, age, gender, assay type, and memory span. Anim Behav 69: 891-898. doi:10.1016/j.anbehav.2004.06.013

Niewalda T, Singhal N, Fiala A, Saumweber T, Wegener S, Gerber B. 2008. Salt processing in larval Drosophila: choice, feeding, and learning shift from appetitive to aversive in a concentration-dependent way. Chem Senses 33: 685-692. doi:10.1093/chemse/bjn037

Michels B, Diegelmann S, Tanimoto H, Schwenkert I, Buchner E, Gerber B. 2005. A role for synapsin in associative learning: the Drosophila larva as a study case. Learn Mem 12: 224-231. doi:10.1101/lm.92805

Michels B, Saumweber T, Biernacki R, Thum J, Glasgow RDV, Schleyer M, Chen Y, Eschbach C, Stocker RF, Toshima N, et al. 2017. Pavlovian conditioning of larval Drosophila: an illustrated, multilingual, hands-on manual for odor-taste associative learning in maggots. Front Behav Neurosci 11: 45. doi: 10.3389/fnbeh.2017.00045

Mishra D, Louis M, Gerber B. 2010. Adaptive adjustment of the generalization-discrimination balance in larval Drosophila. J Neurogenet 24: 168-175. doi:10.3109/01677063.2010.498066

Obermeyer Z, Emanuel EJ. 2016. Predicting the future - Big data, machine learning and clinical medicine. N Engl J Med 375: 1216-1219. doi:10 .1056/NEJMp1606181

Ohl FW, Scheich H, Freeman WJ. 2001. Change in pattern of ongoing cortical activity with auditory category learning. Nature 412: 733-736. doi:10.1038/35089076

Paisios E, Rjosk A, Pamir E, Schleyer M. 2017. Common microbihavioral "footprint" of two distinct classes of conditioned aversion. Learn Mem 24: 191-198. doi:10.1101/lm.045062.117

Pamir E, Szyszka P, Scheiner R, Nawrot MP. 2014. Rapid learning dynamics in individual honeybees during classical conditioning. Front Behav Neurosci 8: 1-17. doi:10.3389/fnbeh.2014.00313

Pauls D, Pfitzenmaier JER, Krebs-Wheaton R, Selcho M, Stocker RF, Thum AS. 2010. Electric shock-induced associative olfactory learning in Drosophila larvae. Chem Senses 35: 335-346. doi:10.1093/chemse/ bjq023

Pech U, Revelo NH, Seitz KJ, Rizzoli SO, Fiala A. 2015. Optical dissection of experience-dependent pre- and postsynaptic plasticity in the Drosophila brain. Cell Rep 10: 2083-2095. doi:10.1016/j.celrep.2015.02.065

Pfeiffer BD, Ngo TT, Hibbard KL, Murphy C, Jenett A, Truman JW, Rubin GM. 2010. Refinement of tolls for targeted gene expression in Drosophila. Genetics 186: 735-755. doi:10.1534/genetics.110.119917

R Development Core Team. 2016. A language and environment for statistical computing. R Foundation for Statistical Computing, Vienna, Austria.

Rescorla RA, Wagner AR. 1972. A theory of Pavlovian conditioning: variations in the effectiveness of reinforcement and nonreinforcement. In Classical conditioning II: current research and theory (ed. Black $\mathrm{AH}$, Prokasy WF), pp.64-99. Appleton-Century-Crofts, New York.

Rohwedder A, Pfitzenmaier JE, Ramsperger N, Apostolopoulou AA, Widmann A, Thum AS. 2012. Nutritional value-dependent and nutritional value-independent effects on Drosophila melanogaster larval behaviour. Chem Senses 37: 711-721. doi:10.1093/chemse/bjs055 
Sang JH, King RC. 1961. Nutritional requirements of axenically cultured Drosophila melanogaster adults. J Exp Biol 38: 793-809.

Saumweber T, Husse J, Gerber B. 2011. Innate attractiveness and associative learnability of odors can be dissociated in larval Drosophila. Chem Senses 36: 223-235. doi:10.1093/chemse/bjq128

Saumweber T, Cano CC, Klessen J, Eichler K, Fendt M, Gerber B. 2014. Immediate and punitive impact of mechanosensory disturbance on olfactory behaviour of larval Drosophila. Biol Open 3: 1005-1010. doi:10 $.1242 /$ bio. 20149183

Saumweber T, Rohwedder A, Schleyer M, Eichler K, Chen Y, Aso Y, Cardona A, Eschbach C, Kobler O, Voigt A, et al. 2018. Functional architecture of reward learning in mushroom body extrinsic neurons of larval Drosophila. Nat Commun 9: 1104. doi:10.1038/ s41467-018-03130-1

Scherer S, Stocker RF, Gerber B. 2003. Olfactory learning in individually assayed Drosophila larvae. Learn Mem 10: 217-225. doi:10.1101/lm .57903

Scheunemann L, Skroblin P, Hundsrucker C, Klussmann E, Efetova M, Schwärzel M. 2013. AKAPS act in a two-step mechanism of memory aquisition. J Neurosci 33: 17422-17428. doi:10.1523/JNEUROSCI $.1303-13.2013$

Schipanski A, Yarali A, Niewalda T, Gerber B. 2008. Behavioural analyses of sugar processing in choice feeding and learning in larval Drosophila. Chem Senses 33: 563-573. doi:10.1093/chemse/bjn024

Schleyer M, Saumweber T, Nahrendorf W, Fischer B, von Alpen D, Pauls D, Thum AS, Gerber B. 2011. A behavior-based circuit model of how outcome expectations organize learned behavior in larval Drosophila. Learn Mem 18: 639-653. doi:10.1101/lm.2163411

Schleyer M, Miura D, Tanimura T, Gerber B. 2015a. Learning the specific quality of taste reinforcement in larval Drosophila. eLife 4: e04711. doi:10.7554/eLife.04711

Schleyer M, Reid SF, Pamir E, Saumweber T, Paisios E, Davies A, Gerber B, Louis M. 2015b. The impact of odor-reward memory on chemotaxis in larval Drosophila. Learn Mem 22: 267-277. doi:10.1101/lm.037978.114

Schleyer M, Fendt M, Schuller S, Gerber B. 2018. Associative learning of stimuli paired and unpaired with reinforcement: evaluating evidence from maggots, flies, bees, and rats. Front Psychol 9: 1494. doi: 10.3389/ fpsyg.2018.01494
Schwärzel M, Müller U. 2006. Dynamic memory networks: dissecting molecular mechanisms underlying associative memory in the temporal domain. Cell Mol Life Sci 63: 989-998. doi:10.1007/s00018-006-6024-8

Takeda K. 1961. Classical conditioned response in the honey bee. J Insect Physiol 6: 168-179. doi:10.1016/0022-1910(61)90060-9

Tedjakumala SR, Giurfa M. 2013. Rules and mechanisms of punishment learning in honey bees: the aversive conditioning of the sting extension response. J Exp Biol 216: 2985-2997. doi:10.1242/jeb.086629

Thum AS, Gerber B. 2018. Connectomics and function of a memory network: the mushroom body of larval Drosophila. Curr Opin Neurobiol 54: $146-154$. doi:10.1016/j.conb.2018.10.007

Trabasso TR. 1963. Stimulus emphasis and all-or-none learning in concept identification. J Exp Psychol 65: 398-406. doi:10.1037/h0042620

Tully T, Quinn WG. 1985. Classical conditioning and retention in normal and mutant Drosophila melanogaster. J Comp Physiol A 157: 263-277. doi:10.1007/BF01350033

Venken KJ, Schulze KL, Haelterman NA, Pan H, He Y, Evans-Holm M, Carlson JW, Levis RW, Spradling AC, Hoskins RA, et al. 2011. MiMIC: a highly versatile transposon insertion resource for engineering Drosophila melanogaster gens. Nat Methods 8: 737-743. doi:10.1038/ nmeth.1662

Vergoz V, Roussel E, Sandoz JC, Giurfa M. 2007. Aversive learning in honeybees revealed by the olfactory conditioning of the sting extension reflex. PLoS One 2: e288. doi:10.1371/journal.pone.0000288

Widmann A, Artinger M, Biesinger L, Boepple K, Peters C, Schlechter J, Selcho M, Thum AS. 2016. Genetic dissection of aversive associative olfactory learning and memory in Drosophila larvae. PLoS Genet 12: e1006378. doi:10.1371/journal.pgen.1006378

Wood ER, Agster KM, Eichenbaum H. 2004. One-trial odor-reward association: a form of event memory not dependent on hippocampal function. Behav Neurosci 118: 526-539. doi:10.1037/0735-7044.118.3 .526

Received December 11, 2018; accepted in revised form February 25, 2019. 


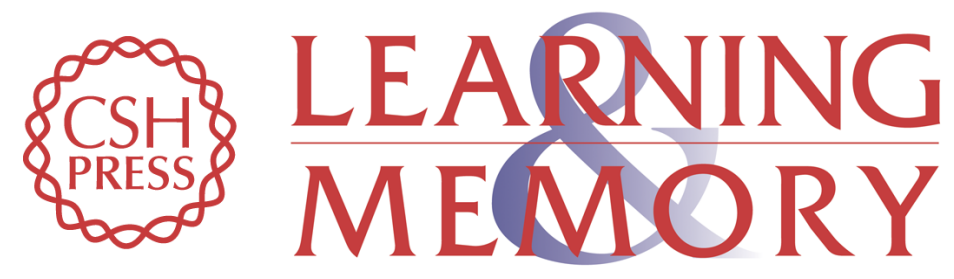

\section{One-trial learning in larval Drosophila}

Alice Weiglein, Florian Gerstner, Nino Mancini, et al.

Learn. Mem. 2019, 26:

Access the most recent version at doi:10.1101//m.049106.118

References This article cites 65 articles, 21 of which can be accessed free at: http://learnmem.cshlp.org/content/26/4/109.full.html\#ref-list-1

Creative This article is distributed exclusively by Cold Spring Harbor Laboratory Press for the Commons License first 12 months after the full-issue publication date (see http://learnmem.cshlp.org/site/misc/terms.xhtml). After 12 months, it is available under a Creative Commons License (Attribution-NonCommercial 4.0 International), as described at http://creativecommons.org/licenses/by-nc/4.0/.

Email Alerting Receive free email alerts when new articles cite this article - sign up in the box at the Service top right corner of the article or click here. 